\title{
Matroids from fuzzy graphs
}

\author{
O.K. Shabna ${ }^{1 *}$ and K. Sameena ${ }^{2}$
}

\section{Abstract}

This paper aims to construct a new class of matroids from fuzzy graphs. Also, basis and circuits for this group of matroids are defined and some of their properties are discussed. The conditions for the existence of weak isomorphism and isomorphism between two matroids from fuzzy graphs are described.

\section{Keywords}

Fuzzy graph, matroids, basis, circuits, weak isomorphism, isomorphism.

\section{AMS Subject Classification} 05C38, 05C72, 05C60, 05C99, 05B35, 05B99.

1,2 Department of Mathematics, MES Mampad College, Malappuram, Kerala-676542, India.

*Corresponding author: ${ }^{1}$ shabnashabiok@gmail.com; ${ }^{2}$ sameenakalathodi@gmail.com

Article History: Received 24 January 2019; Accepted 24 May 2019

\section{Contents}

1 Introduction .................................. 500

1.1 Graph theory . . . . . . . . . . . . . . 500

1.2 Matroid theory . . . . . . . . . . . . . 500

1.3 Fuzzy graph theory . . . . . . . . . . 501

2 Matroids from fuzzy graphs ...................501

2.1 Matroids from fuzzy graphs . . . . . . . . 501

2.2 Basis and circuits for matroid from a fuzzy graph . 502

2.3 Isomorphism of matroids from fuzzy graphs . . 502

3 Conclusion ................................. 503

References. .503

\section{Introduction}

\subsection{Graph theory}

A graph $G$ [2] is an ordered pair $(V(G), E(G))$ consisting of a set $V(G)$ of vertices and a set $E(G)$, disjoint from $V(G)$, of edges, together with an incidence function $\Psi_{G}$ that associates with each edge of $G$ an ordered pair of (not necessarily distinct) vertices of $G$. If $e$ is an edge and $u$ and $v$ are vertices such that $\Psi_{G}(e)=\{u, v\}$, then $e$ is said to join $u$ and $v$, and the vertices $u$ and $v$ are called the ends of $e$. We denote the numbers of vertices and edges in $G$ by $v(G)$ and $e(G)$; these two basic parameters are called the order and size of $G$, respectively.
An edge with identical ends is called a loop, and an edge with distinct ends a link. Two or more links with the same pair of ends are said to be parallel edges. A graph is simple if it has no loops or parallel edges.

A cycle on three or more vertices is a simple graph whose vertices can be arraged in a cyclic sequence in such a way that two vertices are adjacent if they are consecutive in the sequence, and are non-adjacent otherwise; a cycle on one vertex consists of a single vertex with a loop, and a cycle on two vertices consists of two vertices joined by a pair of parallel edges. The length of a cycle is the number of its edges.

An acyclic graph is one that contains no cycles. A connected acyclic graph is called a tree. Acyclic graphs are usually called forests.

Theorem 1.1.1. If $T$ is a tree, then $|E(T)|=|V(T)|-1$

\subsection{Matroid theory}

Matroid theory is an analysis of abstract theory of dependence. In 1935, Whitney coined the word "matroid" in his founding paper entitled "On the abstract properties of linear dependence". Three fundamental properties which are common to linear and algebraic dependence are determined by Van der Waerden in his paper "Morderne Algebra"(1937). Birkhoff(1935) and MacLane(1936, 1938) were also done pioneering works in this area. 
A Matroid [3] $M$ is an ordered pair $(E, \mathscr{I})$ consisting of a finite set $E$ and a collection $\mathscr{I}$ of subsets of $E$ satisfying the following three conditions:

I1. $\phi \in \mathscr{I}$

2. If $I \in \mathscr{I}$ and $I^{\prime} \subseteq I$, then $I^{\prime} \in \mathscr{I}$

I3. If $I_{1}$ and $I_{2}$ are in $\mathscr{I}$ and $\left|I_{1}\right|<\left|I_{2}\right|$, then there is an element $e$ of $I_{2}-I_{1}$ such that $I_{1} \cup e \in \mathscr{I}$

The members of $\mathscr{I}$ are the independent sets of $M$, the subsets of $E$ which are not members of $\mathscr{I}$ are the dependent sets of $M$ and $E$ is the ground set of $M$.

A maximal independent set in $M$ is called a basis or a base of $M$, and we denote the collection of bases of $M$ by $\mathscr{B}$.

A minimal dependent set in an arbitrary matroid $M$ is called a circuit of $M$, and we denote a set of circuits of $M$ by $\mathscr{C}$ or $\mathscr{C}(M)$.

The matroid derived from a graph $G$ is called the cycle matroid or polygon matroid of $G$, denoted by $M(G)$. Clearly a set $X$ of edges is independent in $M(G)$ if and only if $X$ does not contain the edge set of a cycle. A matroid that is isomorphic to the cycle matroid of a graph is called graphic matroid.

Example 1.2.1. Consider the graph $G$ with 4 vertices and 7 edges as shown in Fig 1 . Let $E$ be the edge set of $H$, that is, $\{1,2,3,4,5,6,7\}$, and let $\mathscr{I}$ be the collection of subsets of $E$ that do not contain all of the edges of any simple closed path or cycle of $H$. The cycles of $H$ have edge sets $\{7\},\{5,6\}$, $\{1,2,4\},\{2,3,5\},\{2,3,6\},\{1,3,4,5\}$ and $\{1,3,4,6\}$.

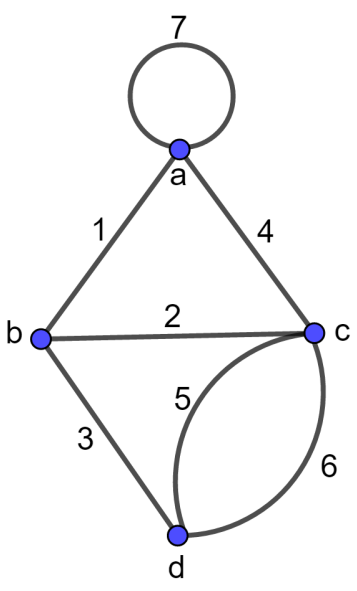

Fig 1.

For this cyclic matroid, the collection of bases,

$$
\begin{aligned}
\mathscr{B}= & \{\{1,2,3\},\{1,2,5\},\{1,2,6\},\{1,3,4\},\{1,3,5\}, \\
& \{1,3,6\},\{1,4,5\},\{1,4,6\},\{2,3,4\},\{2,4,5\}, \\
& \{2,4,6\},\{3,4,5\},\{3,4,6\}\} .
\end{aligned}
$$

Lemma 1.2.1. If $B_{1}$ and $B_{2}$ are bases of a matroid $M$, then $\left|B_{1}\right|=\left|B_{2}\right|$.

\subsection{Fuzzy graph theory}

Foundation for fuzzy graph theory was laid by Rosenfeld in his paper "Fuzzy Graphs", and Yeh and Bang in their paper "Fuzzy graphs, fuzzy relations, and their applications to cluster analysis" in 1975. The first definition of fuzzy graph was given by Kaufman in his paper "Introduction to the Theory of Fuzzy Sets"(1973). Fuzzy analogs of several basic graphtheoretic concepts were introduced by Rosenfeld.

A fuzzy graph [4] $G=(V, \sigma, \mu)$ is a triple consisting of a nonempty set $V$ together with a pair of functions $\sigma: V \longrightarrow$ $[0,1]$ and $\mu: E \longrightarrow[0,1]$ such that for all $x, y \in V, \mu(x y) \leq$ $\sigma(x) \wedge \sigma(y)$.

A path [4] $P$ in a fuzzy graph $G=(V, \sigma, \mu)$ is a sequence of distinct vertices $x_{0}, x_{1}, \ldots x_{n}$ (except possibly $x_{0}$ and $x_{n}$ ) such that $\mu\left(x_{i-1} x_{i}\right)>0, i=1, \ldots, n$.

Here $n$ is called the length of the path.

We call $P$ a cycle if $x_{0}=x_{n}$ and $n \geq 3$.

A fuzzy graph $G=(\sigma, \mu)$ is called a cycle if $(\operatorname{supp}(\sigma), \operatorname{supp}(\mu))$ is a cycle, and $G$ is called a fuzzy cycle if $(\operatorname{supp}(\sigma), \operatorname{supp}(\mu))$ is a cycle and $\nexists$ unique $x y \in \operatorname{supp}(\mu)$ such that $\mu(x y)=\wedge\{\mu(u v) \mid u v \in \sup p(\mu)\}$.

\section{Matroids from fuzzy graphs}

\subsection{Matroids from fuzzy graphs}

Theorem 2.1.1. Let $\bar{G}=(V, \sigma, \mu)$ be a fuzzy graph with correspoding graph $G=(V, E)$ and let

$$
\mathfrak{I}=\{v \subseteq \mu: v \text { does not form any fuzzy cycle }\} .
$$

Then $(\mu, \mathfrak{I})$ form a matroid.

Proof. As an empty set does not contain any fuzzy cycle, $(\mu, \mathfrak{I})$ trivially satisfies axiom I1 .

Let $\xi \in \mathfrak{I}$. That is, $\xi$ does not form any fuzzy cycle. Then, clearly $\xi^{\prime}$ also does not form any fuzzy cycle, $\forall \xi^{\prime} \subseteq \xi$. Thus I 2 is also satisfied by $(\mu, \mathfrak{I})$.

For I3 , assume that $\xi_{1} \cup\{e\} \notin \mathfrak{I}, \forall e \in \xi_{2}-\xi_{1}$. We will show that $\left|\xi_{1}\right| \geq\left|\xi_{2}\right|$. 
Let $e$ be an edge in $\xi_{2}-\xi_{1}$ which connects the vertices $v$ and $w$.

If $\xi_{1} \cup\{e\} \notin \mathfrak{I}$, then the vertices $v, w$ must belong to the same connected component of $\xi_{1}$.

$\Rightarrow V\left(\xi_{1}\right)=V\left(\xi_{2}\right)$, where $V\left(\xi_{1}\right)$ and $V\left(\xi_{2}\right)$ are the vertex sets of $\xi_{1}$ and $\xi_{2}$ respectively, and each connected component of $\xi_{2}$ is a subgraph of a connected component of $\xi_{1}$.

Which means that

$$
k_{\xi_{2}} \geq k_{\xi_{1}}
$$

where $k_{\xi_{1}}$ and $k_{\xi_{2}}$ are the number of connected components in $\xi_{1}$ and $\xi_{2}$ respectively.

We have, by Theorem 1.1.1, $\left|\xi_{1}\right|=\left|V\left(\xi_{1}\right)\right|-k_{\xi_{1}}$ and $\left|\xi_{2}\right|=\left|V\left(\xi_{2}\right)\right|-k_{\xi_{2}}$, thus combining with (2.1), we have $\left|\xi_{1}\right| \geq\left|\xi_{2}\right|$, which is a contradiction.

Example 2.1.1. Cosider the fuzzy graph $G=(V, \sigma, \mu)$; where $V=\{p, q, r, s\}$. Let $X=\{p q, q r, r s, s p\}$. Let $\sigma(x)=1, \forall x \in V$ and let $\mu$ be the fuzzy subset of $X$ defined by $\mu(p q)=\mu(s r)=$ 0.3 , and $\mu(q r)=\mu(s p)=0.2$.

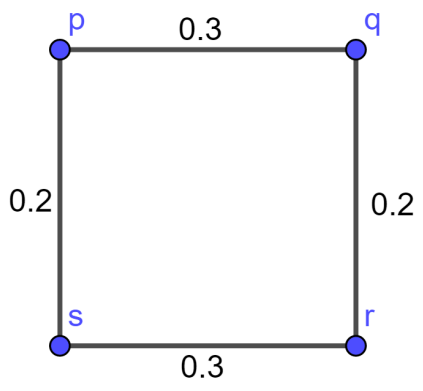

Fig 2.

Here $\{p q, q r, r s, s p\}$ is the only fuzzy cycle, so let $\mathfrak{I}=\{v \subseteq \mu:|v| \leq 3\}$. Then $(\mu, \mathfrak{I})$ matroid on the given fuzzy graph $G$.

If we redefine the above fuzzy graph $G=(V, \sigma, \mu)$ by $\mu(q r)=0.1$

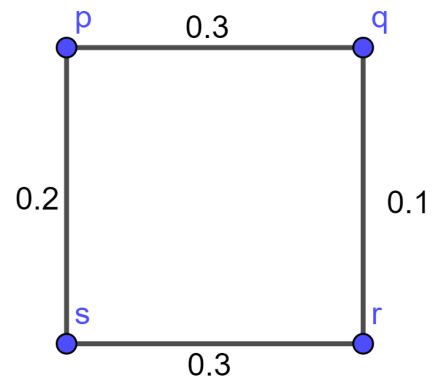

Fig 3.

Here any of subsets of $\mu$ does not contain a fuzzy cycle. Thus $\mathfrak{I}=\{v: v \subseteq \mu\}=2^{\mu}$, and $(\mu, \mathfrak{I})$ form a matroid on $G$.

Remark 2.1.1. A subset of a fuzzy edge set corresponding to a dependent set in the matroid of crisp graph need not be dependent in the corresponding matroid from the fuzzy graph.

\subsection{Basis and circuits for matroid from a fuzzy graph}

Definition 2.2.1. Let $\bar{M}=(\mu, \mathfrak{I})$ be a matroid from a fuzzy graph $G=(V, \sigma, \mu)$. The maximal independent subsets of $\mu$ is referred as basis of $\bar{M}$.

The collection of bases, denoted by $\mathscr{B}(E)$, is given by

$$
\mathscr{B}(E)=\{v \subseteq \mu: v \in \mathfrak{I}, v \cup\{e\} \notin \mathfrak{I}, \forall e \in \mu-v\}
$$

Lemma 2.2.1. If $B$ and $B^{\prime}$ are bases of a matroid $\bar{M}$ from a fuzzy graph $G=(V, \sigma, \mu)$, then $|B|=\left|B^{\prime}\right|$.

Proof. Let $\bar{M}=(\mu, \mathfrak{I})$ be a matroid from a fuzzy graph $G=$ $(V, \sigma, \mu)$, and let $B, B^{\prime} \in \mathscr{B}(E)$. Suppose that $|B|>\left|B^{\prime}\right|$. Then, as both $B$ and $B^{\prime}$ are in $\mathfrak{I}$, by I(3), there is an element $e \in$ $B-B^{\prime}$ such that $B^{\prime} \cup\{e\} \in \mathfrak{I}$. This is a contradiction to the maximal independence of $B^{\prime}$. Thus $|B| \leq\left|B^{\prime}\right|$. Similarly, we can show $\left|B^{\prime}\right| \leq|B|$. Hence $|B|=\left|B^{\prime}\right|$.

Definition 2.2.2. Let $\bar{M}=(\mu, \mathfrak{I})$ be a matroid from a fuzzy graph $G=(V, \sigma, \mu)$. The minimal dependent subset of $\mu$ will be called circuit of $\bar{M}$.

The collection of circuits, denoted by $\mathscr{C}(\mu)$, is given by

$$
\mathscr{C}(\mu)=\{\varphi \subseteq \mu: \varphi \notin \mathfrak{I}, \varphi-\{x\} \in \mathfrak{I}, \forall x \in \varphi\}
$$

Theorem 2.2.1. Let $\mathscr{C}(\mu)$ be the collection of circuits of a matroid $\bar{M}=(\mu, \mathfrak{I})$ from a fuzzy graph $G=(V, \sigma, \mu)$. Then the following are satisfied:

(i) $\phi \notin \mathscr{C}(\mu)$

(ii) If $\varphi, \varphi^{\prime} \in \mathscr{C}(\mu)$, and $\varphi \subseteq \varphi^{\prime}$, then $\varphi=\varphi^{\prime}$.

Proof. Let $\mathscr{C}(\mu)$ be the collection of circuits of a matroid $\bar{M}=(\mu, \mathfrak{I})$ from a fuzzy graph $G=(V, \sigma, \mu)$.

By I(1), we have $\phi \in \mathfrak{I}$, which follows $\phi \notin \mathscr{C}(\mu)$.

For $(i i)$, suppose $\varphi \neq \varphi^{\prime}$. Then $\varphi^{\prime}$ is not minimally dependent, contradicts the fact $\varphi^{\prime} \in \mathscr{C}(\mu)$. Thus $\varphi=\varphi^{\prime}$.

\subsection{Isomorphism of matroids from fuzzy graphs}

Definition 2.3.1. Let $\bar{M}_{1}=\left(\mu_{1}, \mathfrak{I}_{1}\right)$ and $\bar{M}_{2}=\left(\mu_{2}, \mathfrak{I}_{2}\right)$ be two matroids from the fuzzy graphs $G_{1}=\left(V_{1}, \sigma_{1}, \mu_{1}\right)$ and $G_{2}=\left(V_{2}, \sigma_{2}, \mu_{2}\right)$ respectively. $\bar{M}_{1}$ and $\bar{M}_{2}$ are said to be weak isomorphic if the following conditions are satisfied:

$i$. There is a bijection $\psi$ from $\mu_{1}$ to $\mu_{2}$ such that for all $v \subseteq \mu_{1}, v \in \mathfrak{I}_{1}$ if and only if $\psi(v) \in \mathfrak{I}_{2}$

ii. There is a bijection $\phi: V_{1} \longrightarrow V_{2}$ such that for all $x \in V_{1}$, $\sigma_{1}(x)=\sigma_{2}(\phi(x))$, and $\mu_{1}(x, y) \leq \mu_{2}(\phi(x), \phi(y))$, $\forall x, y \in V_{1}$.

Remark 2.3.1. A weak isomorphism preserves the structure of the matroid and the weights of the vertices but not necessarily the weights of the edges. 
Example 2.3.1. Let $\bar{M}_{1}=\left(\mu_{1}, \mathfrak{I}_{1}\right)$ and $\bar{M}_{2}=\left(\mu_{2}, \mathfrak{I}_{2}\right)$ be two matroids from the fuzzy graphs $G$ and $G^{\prime}$ with underlying sets $V$ and $V^{\prime}$ respectively with $V=\{a, b, c\}$ and $V^{\prime}=\left\{a^{\prime}, b^{\prime}, c^{\prime}\right\}$ as in Fig. 4.

Here $\mathfrak{I}=2^{E}$ and $\mathfrak{I}^{\prime}=2^{E}$.

Here

$$
\begin{aligned}
\mathfrak{I}_{1}= & \{\phi,\{a b\},\{b c\},\{c d\},\{a b, b c\},\{a b, c d\},\{b c, c d\}, \\
& \{a b, b c, c d\}\}
\end{aligned}
$$

and

$$
\begin{aligned}
\mathfrak{I}_{2}= & \left\{\phi,\left\{a^{\prime} b^{\prime}\right\},\left\{b^{\prime} c^{\prime}\right\},\left\{c^{\prime} d^{\prime}\right\},\left\{a^{\prime} b^{\prime}, b^{\prime} c^{\prime}\right\},\left\{a^{\prime} b^{\prime}, c^{\prime} d^{\prime}\right\},\right. \\
& \left.\left\{b^{\prime} c^{\prime}, c^{\prime} d^{\prime}\right\}\left\{a^{\prime} b^{\prime}, b^{\prime} c^{\prime}, c^{\prime} d^{\prime}\right\}\right\}
\end{aligned}
$$

Then clearly, we can form a bijection from $\mathfrak{I}_{1} \longrightarrow \mathfrak{I}_{2}$.

Now defining and $\phi: V \longrightarrow V^{\prime}$ as follows:

$\phi(a)=a^{\prime}, \phi(b)=b^{\prime}, \phi(c)=c^{\prime}$; this $\phi$ is a bijective map satisfying

$\sigma(v)=\sigma^{\prime}(\phi(v)), \forall v \in V$ and $\mu(u, v) \leq \mu^{\prime}(\phi(u), \quad \phi(v))$,

$\forall u, v \in V$.

Thus $\bar{M}_{1}$ is weak isomorphic to $\bar{M}_{2}$.
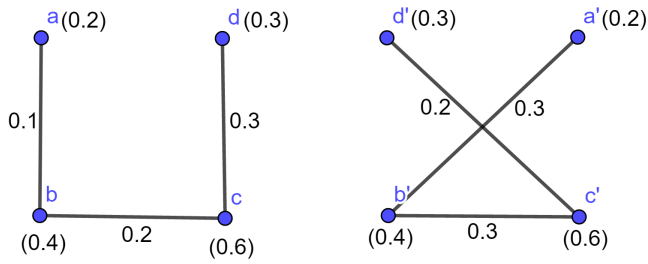

Fig. 4

Definition 2.3.2. Let $\bar{M}_{1}=\left(\mu_{1}, \mathfrak{I}_{1}\right)$ and $\bar{M}_{2}=\left(\mu_{2}, \mathfrak{I}_{2}\right)$ be two matroids from the fuzzy graphs $G_{1}=\left(V_{1}, \sigma_{1}, \mu_{1}\right)$ and $G_{2}=\left(V_{2}, \sigma_{2}, \mu_{2}\right)$ respectively. $\bar{M}_{1}$ and $\bar{M}_{2}$ are said to be isomorphic, denoted by $\bar{M}_{1} \cong \bar{M}_{2}$, if the following conditions are satisfied:

i. There is a bijection $\psi$ from $\mu_{1}$ to $\mu_{2}$ such that for all $v \subseteq \mu_{1}, v \in \mathfrak{I}_{1}$ if and only if $\psi(v) \in \mathfrak{I}_{2}$

ii. There is a bijection $\phi: V_{1} \longrightarrow V_{2}$ such that for all $x \in V_{1}$, $\sigma_{1}(x)=\sigma_{2}(\phi(x))$, and $\mu_{1}(x, y)=\mu_{2}(\phi(x), \phi(y))$, $\forall x, y \in V_{1}$.

Remark 2.3.2. An isomorphism preserves the structure of the matroid, the weights of the vertices and the weights of the edges.

Example 2.3.2. Consider the matroids $\bar{M}_{1}=\left(\mu_{1}, \mathfrak{I}_{1}\right)$ and $\bar{M}_{2}=\left(\mu_{2}, \mathfrak{I}_{2}\right)$ derived from the two fuzzy graphs given in Fig.5 with underlying sets $V$ and $V^{\prime}$ respectively as $V=$ $\{a, b, c, d\}$ and $V^{\prime}=\left\{a^{\prime}, b^{\prime}, c^{\prime}, d^{\prime}\right\}$.

Here, the structure, weights of vertices and weights of edges of $\bar{M}_{1}$ and $\bar{M}_{2}$ are same.

That is, $\bar{M}_{1} \cong \bar{M}_{2}$.
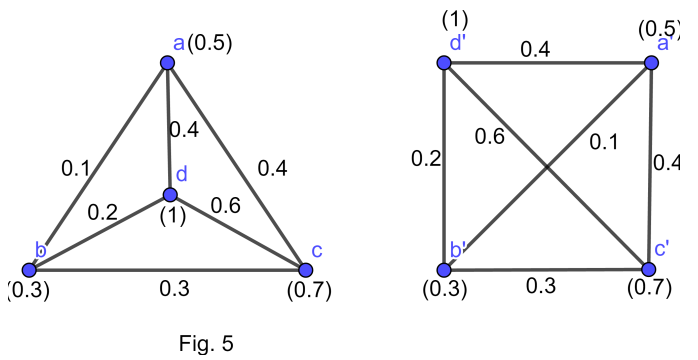

\section{Conclusion}

In this paper, We have constructed a new class of matroids from fuzzy graphs. Let $G$ be a fuzzy graph, then the maximal independent subset and minimal dependent subset of the edge set of underlying graph of $G$ form a basis and circuit respectively, for the matroid from the fuzzy graph $G$. If there is a weak isomorphism between two matroids of this kind, then there will preserve the structure of the matroid and weights of vertices, but the weights of edges need not be preserved. While, if they are isomorphic, then they will preserve structure, weights of vertices as well as weights of edges.

\section{Acknowledgement}

The work of the first author is supported by the "CSIR, Human Resource and Development Group, India, under the grant number 08/706(0002)/2018-EMR-I Dated 24/04/2019".

\section{References}

[1] Leonidas S. Pitsoulis, Topics in Matroid Theory, Springer , 2014.

[2] J.A Bondy and V.S.R Murty, Graph Theory, Springer, 2007.

[3] James Oxley, Matroid Theory, Oxford Uiversity Press, 1992.

[4] Sunil Mathew, John N. Mordeson and Davender S. Malik, Fuzzy Graph Theory, Springer, 2018.

[5] K. Truemper, Matroid Decomposition, Leibniz, 1998.

[6] Wei Yao and Fu-Gui Shi, Bases axioms and circuits axioms for fuzzifying matroids, Fuzzy Sets and Systems 161(2010), 3155-3165.

[7] Chun-E Huang, Graphic and representable fuzzifying matroids, Proyecciones Journal of Mathematics 29(2010), $17-30$.

${ }^{[8]}$ L. X. Lu and W. W. Zheng, Categorical relations among matroids, Fuzzy matroids and Fuzzifying matroids, Iranian Journnal of Fuzzy systems 7(2010), 81-89.

[9] Yonghong Li, Yingjie Shi and Dong Qiu, The Research of the closed fuzzy matroids, Journal of Mathematics and Informatics, 2 (2014), 17-23.

[10] Talal Al- Hawary, Fuzzy closure matroids, Matematika 32(2016), 69-74.

[11] John N. Mordeson, Bases of Fuzzy Vector Space, Information Sciences 67(1993), 87-92. 
[12] Fu-Gui Shi and Chun-E Haung, Fuzzy bases and the fuzzy dimension of fuzzy vector spaces, Mathematical Communications 1,15(2010), 303-310.

[13] A. Nagoor Gani, J. Malarvizhi, Isomorphism on fuzzy graphs, International Journal of Mathematical and Computational Sciences 2(2008), 825-831.

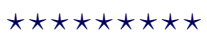

ISSN(P):2319-3786

Malaya Journal of Matematik

ISSN(O):2321 - 5666

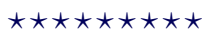

